\title{
Fungos Filamentosos Associados às Espécies Atta sexdens (Linnaeus) e Atta laevigata (F. Smith) (Hymenoptera: Formicidae)
}

\author{
Aline Silvestre Pereira Dornelas, Renato de Almeida Sarmento, Gil Rodrigues dos Santos, \\ Mariela Otoni do Nascimento \& Danival José de Souza
}

Universidade Federal do Tocantins, e-mail: lyne_silvestre@hotmail.com, rsarmento@uft.edu.br, gilrsan@uft.edu.br, marielaotoni@gmail.com, danival@uft.edu.br (Autor para correspondência ${ }^{\varpi}$ ).

\section{EntomoBrasilis 9 (1): 26-30 (2016)}

Resumo. As formigas-cortadeiras, Atta e Acromyrmex, são consideradas as principais pragas no sistema agroflorestal da Região Neotropical. Isso porque cortam material vegetal que servirá de alimento ao fungo simbionte que elas cultivam. Diversos fungos, que ocorrem em condições naturais no solo, podem ser encontrados associados às formigas-cortadeiras, muitos deles comprovadamente entomopatogênicos. No entanto, esses agentes não têm sido usados em programa de controle biológico dessas formigas. O objetivo deste estudo foi isolar e identificar fungos filamentosos associados às operárias de Atta sexdens e Atta laevigata e testar sua patogenicidade contra operárias da formiga A. sexdens. Para isolamento dos fungos, foi coletado um total de 180 operárias forrageadoras em seis colônias (30 por colônia), sendo 90 de $A$. sexdens e 90 de A. laevigata. Dentre os fungos isolados das operárias de A. sexdens, foram identificados Metarhizium anisopliae (Metchnikoff) Sorokin, Aspergillus flavus Link, Acremonium sp. 1, Aspergillus sp.1, Colletotrichum sp. e Acremonium sp. 2. Os fungos encontrados em associação com as operárias da A. laevigata foram Mucor sp., Aspergillus flavus, Fusarium solani (Martius) Saccardo, A. niger van Tieghem. A patogenicidade de três desses fungos (A. flavus, A. niger e $M$. anisopliae) foi testada em operárias de A. sexdens. Nos testes com A. niger e M. anisopliae, o tempo para causar mortalidade de 50\% das operárias foi de cinco dias $\left(\mathrm{TL}_{50}=5\right.$ dias) e, nos dois casos, o TL foi significativamente inferior ao tratamento controle. Assim, testes devem prosseguir com esses isolados que apresentam potencial no uso do controle biológico das formigas-cortadeiras.

Palavras-chave: Aspergillus; Controle biológico; Entomopatógenos; Metarhizium; Simbiose.

\section{Filamentous Fungi Associated With Atta sexdens (Linnaeus) and Atta laevigata (F. Smith) (Hymenoptera: Formicidae)}

Abstract. Leaf-cutting ants, Atta and Acromyrmex genera, are considered major pests in a neotropical agroforestry system because they cut plant material that will serve as food for the symbiotic fungus cultivated by them. Several fungi naturally occurring in the soil can be found associated with leaf-cutting ants, many of them are demonstrably entomopathogenic. However, these agents have not been used as biological control of leaf-cutting ants. The aim of this study was to isolate and to identify filamentous fungi associated with forage workers of Atta sexdens (Linnaeus) and Atta laevigata (F. Smith) and to test their pathogenicity against workers from laboratory colonies. To isolate filamentous fungi, it was collected a total of 180 forage workers (30 in each colony) in six field colonies, 90 of $A$. sexdens and 90 workers of $A$. laevigata. Six fungi species from $A$. sexdens were isolated and identified: Metarhizium anisopliae (Metchnikoff) Sorokin, Aspergillus flavus Link, Acremonium sp. 1, Aspergillus sp. 1, Colletotrichum sp. and Acremonium sp. 2. In A. laevigata, it was found four species: Mucor sp., Aspergillus flavus, Fusarium solani (Martius) Saccardo, Aspergillus niger van Tieghem. Three of these fungi were selected for pathogenicity tests against workers of the leaf-cutting ant A. sexdens: A. flavus, A. niger and $M$. anisopliae. The LT (time to cause $50 \%$ mortality of workers) in tests with $A$. niger and $M$. anisopliae were five days and significantly lower than the control group. Therefore, further tests should proceed with those isolates to demonstrate their potential use in the biological control of leafcutting ants.

Keywords: Aspergillus; Biological control; Entomopathogens; Metarhizium; Symbiosis.

$\mathrm{s}$ formigas cultivadoras de fungo pertencem à tribo Attini e são exclusivas do Novo Mundo, ocorrendo dos Estados Unidos ao Norte da Argentina. Atualmente, são conhecidos 16 gêneros, sendo que novos gêneros foram descritos recentemente (Kuingenberg \& Brandão 2009; Sosa-Calvo et al. 2013). Essas formigas se especializaram em uma dieta essencialmente fungívora (SchULTZ \& BRADY 2008).

Dentre as formigas cultivadoras de fungo, destacam-se as cortadeiras, aquelas pertencentes aos gêneros Atta e Acromyrmex, conhecidas popularmente como saúvas e quenquéns, respectivamente. Elas recebem esse nome por cortarem e carregarem para o interior dos ninhos partes frescas de plantas. Várias espécies vegetais são cortadas, tanto na fase de brotação quanto na fase adulta, e esses fragmentos vegetais servem como substrato para o desenvolvimento do fungo simbionte Leucoagaricus gongylophorus (Möller) Singer (Basidiomycota:
Agaricales: Lepiotaceae) do qual elas se alimentam. O fungo simbionte mutualista se desenvolve no interior da colônia sobre o substrato vegetal processado pelas operárias, sendo o conjunto fungo-substrato comumente chamado de jardim de fungo ou, ainda, esponja fúngica em referência a seu aspecto. Uma vez que algumas espécies dos gêneros Atta e Acromyrmex são consideradas as principais pragas agrícolas e florestais na região Neotropical, existe uma preocupação constante com o seu controle em vários agroecossistemas (DELLA LuCia et al. 2014). A forma mais frequentemente utilizada para o controle desses insetos é por meio do método químico com predominância do uso de iscas tóxicas, devido ao baixo custo e eficiência demonstrada (JACCOUD 2000). 
Questões econômicas e ambientais têm pressionado as empresas do setor florestal a melhorarem o rendimento das técnicas de controle químico e incentivado a experimentação de novas tecnologias de controle que não venham a degradar o meio ambiente. Neste contexto, as pesquisas científicas têm buscado o desenvolvimento de alternativas para o controle de formigascortadeiras, a exemplo de micro-organismos, merecendo destaque os fungos entomopatogênicos (DIEHL-FLEIG et al. 1988; Ribeiro et al. 2012).

Oobjetivodestetrabalhofoiisolar eidentificarfungos filamentosos associados com as formigas-cortadeiras Atta sexdens (Linnaeus) e Atta laevigata (F. Smith), e testar a patogenicidade de alguns isolados (Aspergillus flavus Link, Aspergillus niger (van Tieghem) e Metarhizium anisopliae (Metchnikoff) Sorokin, fornecendo assim subsídios para que estes agentes possam ser usados em futuros programas de controle biológico.

\section{MATERIAL E MÉTODOS}

Coleta de operárias. Foram coletadas operárias forrageadoras de $A$. sexdens e A. laevigata ( $3 \mathrm{~mm}$ delargura de cápsula cefálica) em fevereiro de 2013, oriundas de três colônias de cada espécie, totalizando seis. Estas estavam localizadas na Universidade Federal do Tocantins, Campus de Gurupi, numa altitude de 287 metros, latitude $11^{\circ} 43^{\prime} 45^{\prime \prime}$ Sul e longitude $49^{\circ} 04^{\prime} 07^{\prime \prime}$ Oeste. Pelo tamanho das colônias, atividade das trilhas de forrageamento, número de olheiros ativos e volume de terra solta, todas as colônias possuíam, muito provavelmente, idade superior a três anos (Grandeza et al. 1999).

Foram coletadas 30 operárias de cada colônia, as quais foram acondicionadas em recipientes esterilizados até serem levadas ao laboratório. No total, foram utilizadas 90 operárias de cada espécie de formiga, perfazendo um total de 180 . Em sala climatizada, as operárias foram colocadas individualmente em placas de Petri de $(\varnothing=9 \mathrm{~cm})$ e supridas diariamente com água e mel, conforme metodologia usada em trabalho com $A$. bisphaerica (Ribeiro et al. 2012). Inspeções diárias para verificar morte de operárias foram realizadas até $010^{\circ}$ dia. As operárias mortas foram lavadas em soluções seriadas de hipoclorito de sódio 4\%, álcool 70\% e água destilada autoclavada. Posteriormente, foram transferidas para microtubos de centrifugação, estéreis, contendo algodão umedecido, sendo mantidas em sala com temperatura de $25 \pm$ $2{ }^{\circ} \mathrm{C}, 70 \pm 2,5 \%$ U.R. e fotofase de 12 horas até que as formigas apresentassem extrusão de fungos.

Isolamento dos Fungos. Após a extrusão dos fungos nos cadáveres das formigas-cortadeiras, foram realizadas repicagens em ambiente estéril até a obtenção do isolado puro. Os fungos repicados foram cultivados em placas de Petri $(\varnothing=9 \mathrm{~cm})$ em meio de cultura BDA (batata, dextrose, ágar) $\operatorname{com~} \mathrm{pH}=6,2$, acrescido de antibiótico cloranfenicol (o,05 g/L) (ALVES \& FARIA 2010). Para o crescimento dos fungos, as placas foram mantidas em sala com temperatura de $25 \pm 2{ }^{\circ} \mathrm{C}$, umidade relativa $70 \pm 2,5$ $\%$ e fotofase de 12 horas.

Identificação dos Fungos. Os fungos foram identificados no laboratóriodeFitopatologia daUniversidadeFederaldoTocantins, Campus de Gurupi e no Laboratório de Ecologia e Sistemática de Fungos, Departamento de Bioquímica e Microbiologia da Universidade Estadual Paulista - UNESP - São Paulo. A identificação foi realizada segundo caracteres morfológicos, principalmente conídios e conidióforos, encontrados em tratados taxonômicos clássicos e com o auxílio de microscopia ótica (SAMSON 2000; Domsch et al. 2007). Adicionalmente, literaturas específicas foram utilizadas para a identificação dos fungos (Alexopoulos et al. 1996; BARNetT \& HunTER 1998).

Infecção de operárias de $A$. sexdens. Avaliou-se a patogenicidade de três isolados contra operárias de $A$. sexdens: A. flavus, A. niger e $M$. anisoplae. Foram testadas apenas operárias de $A$. sexdens, pois esta era a única colônia mantida em laboratório com tamanho ideal ( $3 \mathrm{~L}$ de jardim de fungo) e número suficiente de operárias médias $(\sim 3 \mathrm{~mm}$ de largura de cápsula cefálica). As operárias foram retiradas sempre do interior da colônia de forma a diminuir variações quanto à idade das mesmas, uma vez que há uma tendência de operárias internas serem as mais jovens da colônia (WILSON 1971). A escolha dos fungos se deu por suas prevalências nos nossos estudos e pela alta produção de conídios em laboratório (análise visual da colônia em placa de Petri). Primeiramente, foram aplicados no tórax $1 \mu \mathrm{L}$ de suspensão de conídios ( $1 \times 10^{7}$ conídios x mL $\left.{ }^{-1}\right)$ de $A$. flavus, A. niger ou $M$. anisopliae, preparada com água destilada e 0,05 \% do agente surfactante Tween-80. A concentração de conídios foi estabelecida a partir de testes preliminares com diferentes concentrações. Os tratamentos consistiram de: (1) inoculação das operárias com suspensão de A. flavus, (2) inoculação nas operárias com suspensão de $A$. niger, (3) inoculação nas operárias com suspensão de $M$. anisopliae, (4) aplicação de água + Tween80 no tórax das operárias como controle. Foram utilizadas 20 operárias de cada espécie para cada tratamento. Os testes de mortalidade com cada isolado foram realizados em semanas diferentes, daí ter se repetido o controle para cada um deles.

Após as aplicações, as operárias foram transferidas individualmente para placas de Petri $(\varnothing=9 \mathrm{~cm})$ e supridas com água e mel. Elas foram mantidas em sala climatizada $\left(25^{\circ} \mathrm{C} \pm 2\right.$, $70 \pm 2,5 \%$ de Umidade Relativa U.R. e 12 horas de fotofase). As formigas foram monitoradas até o décimo dia após o início do experimento.

A fim de confirmar a causa da mortalidade das operárias nesse teste, procedeu-se ao isolamento dos fungos testados a partir das operárias mortas. Para isso, foi utilizado o mesmo protocolo de isolamento desses a partir das operárias forrageadoras do campo.

Análises estatísticas. Curvas de sobrevivência foram geradas como uma função do tempo de observação da morte por meio do método de Kaplan-Meier (KAPLAN \& MeIER 1958) e comparadas duas a duas pelo teste de Log-Rank (Mantel 1966; Peto \& Peto 1972) a $P<0,05$ para verificar as diferenças entre os grupos de tratamentos. Para a estimativa de $\mathrm{TL}_{50}$ (tempo letal para morte de 50\% dos indivíduos testados) foi utilizada a análise de probit (FInNEY 1952). Todas as análises foram feitas com o auxílio do programa Statistica 7.0 (STATSOFT 2004).

\section{RESULTADOS E DISCUSSÃO}

Das 90 operárias da espécie $A$. sexdens coletadas, 23 (25,6\%) apresentaram esporulação do fungo após a sua morte, a qual foi verificada até o décimo quinto dia. Da primeira colônia, quatro formigas apresentaram esporulação de fungos, sendo que uma dessas operárias apresentou dois tipos de fungos (M. anisopliae e A. flavus). Na segunda colônia, nove operárias exibiram esporulação de fungos no seu exoesqueleto e, novamente, dois fungos diferentes esporularam numa operária ( $M$. anisopliae e A. flavus). Já a terceira colônia, foi a que apresentou maior número de esporulação de fungo, com um total de dez operárias. Dentre os fungos isolados nas operárias, foram identificadas seis espécies de fungos associados à $A$. sexdens (Tabela 1), com predomínio de $M$. anisopliae (17\% de operárias infectadas). Este último é um fungo interessante, pois tem se mostrado patogênico para operárias de $A$. sexdens em testes de laboratório (DIEHL-FLEIG et al. 1988) e considerado promissor no controle de formigas e outros insetos (JACCOUd et al. 1999; LoPEZ \& ORduZ 2003; HugheS \& BoOmsma 2004; Ferraz et al. 2008; MaUrya et al. 2011).

Em A. laevigata, constatou-se que 20 (22,2\%) operárias tiveram a presença de fungos em seu exoesqueleto. Este valor é bem próximo aos encontrado para $A$. sexdens e reflete a frequência de fungos filamentosos associados a operárias forrageadoras no campo, para as condições e local do estudo. Em A. bisphaerica, a frequência de fungos filamentosos isolados de forrageadoras 
foi um pouco maior: 30\% (Ribeiro et al. 2012). A primeira colônia apresentou esporulação em três operárias de apenas um tipo de fungo nas operárias. Na segunda colônia, 11 formigas apresentaram fungos, sendo que uma destas apresentou a esporulação de dois fungos no seu exoesqueleto (Mucor sp. e $A$. niger). Na terceira colônia, as operárias apresentaram a esporulação de apenas um tipo de fungo (A. niger). No total, quatro espécies de fungos foram identificadas em associação com A. laevigata, sendo Mucor sp.1 e A. niger as mais prevalentes ( $8 \%$ cada). Nenhuma espécie tipicamente entomopatogênica, como M. anisopliae, foi isolada nas forrageadoras de A. laevigata.

Nos experimentos para testar a virulência de alguns isolados, o grupo de operárias de $A$. sexdens que recebeu aplicação do fungo A. flavus apresentou $\mathrm{TL}_{50}=12,9$ dias. No grupo controle, encontrou-se $\mathrm{TL}_{50}=30,6$ dias; apenas três operárias morreram até o décimo dia de avaliação (Figura 1A). Apesar das curvas de mortalidade do tratamento e controle diferirem estatisticamente (Teste de Log-Rank, $P=0,024$ ), a virulência desse isolado se mostrou baixa, nessa concentração de conídios, considerando-se os valores obtidos de $\mathrm{TL}_{50}$.
$\mathrm{O}$ grupo que recebeu uma suspensão $1 \times 10^{7}$ conídios $\times \mathrm{mL}^{-1}$ do fungo $A$. niger apresentou $\mathrm{TL}_{50}=4,50$ dias. Foi verificado que $95 \%$ das operárias do grupo tratamento morreram até o décimo dia de avaliação, ao passo que o tratamento controle apresentou uma mortalidade de $35 \%$ até o décimo dia (Figura 1B) e apresentou $\mathrm{TL}_{50}=8,83$ dias. Essa diferença entre as curvas de sobrevivência de tratamento e controle foi estatisticamente significativa (Teste de Log-Rank, $P<0,001)$. As operárias que receberam o tratamento com o fungo $M$. anisopliae apresentaram $\mathrm{TL}_{50}=3,8$ dias e todas morreram até $\mathrm{o} 10^{\circ}$ dia após o início dos experimentos. O grupo controle apresentou $\mathrm{TL}_{50}=15,8$ dias e apenas $25 \%$ das operárias morreram até o $10^{\circ}$ dia. As curvas de sobrevivência dos grupos tratamento e controle diferiram estatisticamente (Teste de LogRank, $P<0,001$; Figura 1C).

As formigas se contaminaram com os fungos registrados em nosso estudo, muito provavelmente, quando se alimentaram de material vegetal contendo esses fungos. Alguns dos gêneros de fungos encontrados podem ser endofíticos. Por exemplo, o gênero Acremonium possui espécies endofíticas, podendo, inclusive, estar associadas com mecanismos de defesa das plantas contra insetos (BREEN 1994; RoDRIGUEZ et al. 2009). Outra

Tabela 1. Número total de operárias infectadas de acordo com a espécie de fungo e de formiga-cortadeira.

\begin{tabular}{|c|c|c|}
\hline \multirow{2}{*}{ Táxon } & \multicolumn{2}{|c|}{$\mathrm{N}^{0}$ de operárias infectadas } \\
\hline & Atta sexdens & Atta laevigata \\
\hline Acremonium sp.1 & 1 & - \\
\hline Acremonium sp.2 & 1 & - \\
\hline Aspergillus flavus & 3 & 1 \\
\hline Aspergillus niger & - & 8 \\
\hline Aspergillus sp.1 & 2 & - \\
\hline Colletotrichum sp.1 & 1 & - \\
\hline Fusarium solani & - & 3 \\
\hline Metarhizium anisopliae & 15 & - \\
\hline Mucor sp.1 & - & 8 \\
\hline
\end{tabular}

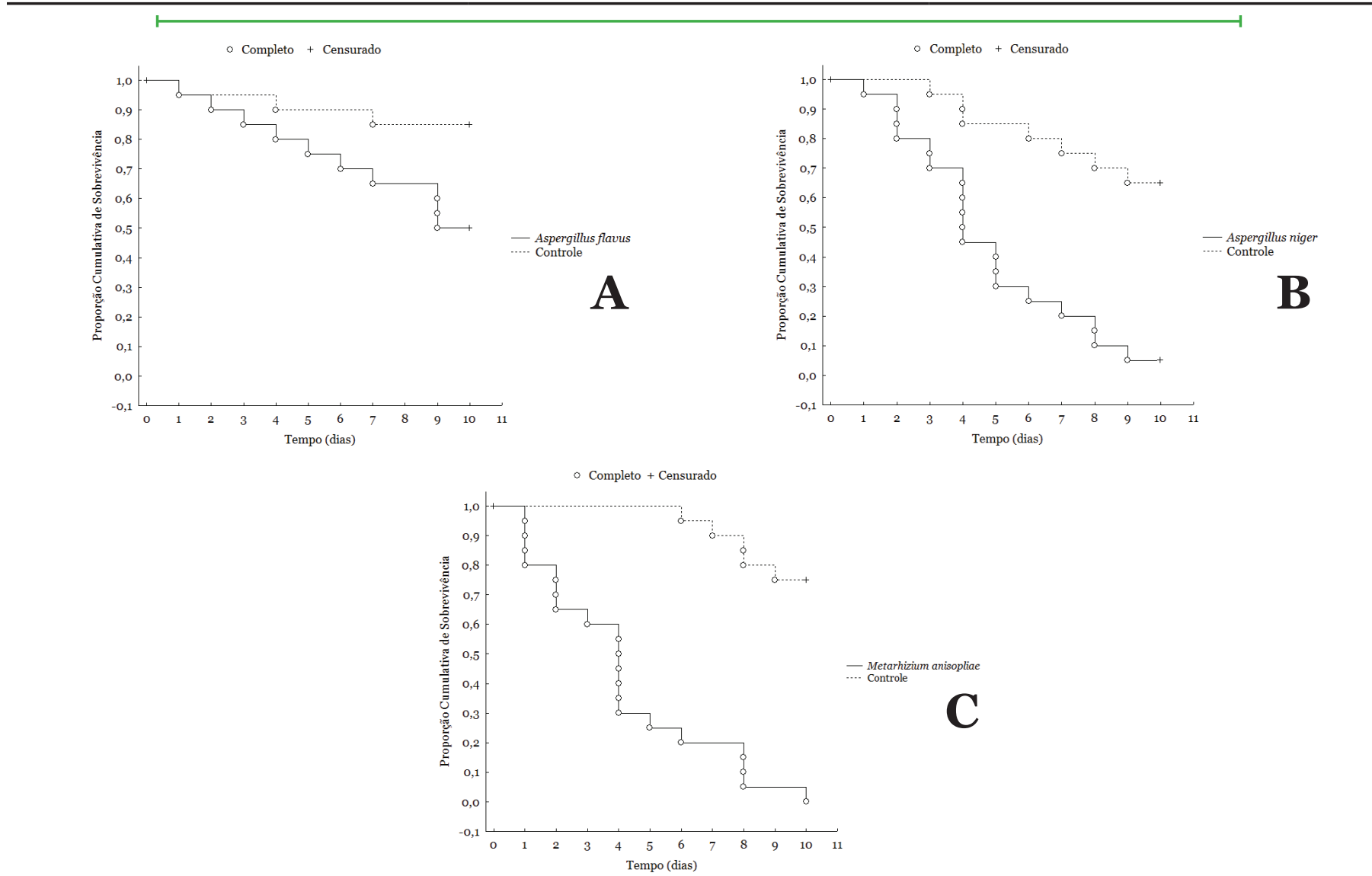

Figura 1. Curvas de sobrevivência de Kaplan-Meier de operárias de Atta sexdens submetidas aos tratamentos (linha contínua) com os fungos Aspergillus flavus (A), Aspergillus niger (B) ou Metarhizium anisopliae (C), todos na concentração de 1 x $10^{7}$ conídios x mL-1; linha tracejada indica formigas que receberam água com solução de Tween-80 0,05\%. 
forma de contaminação das formigas seria pelo contato das operárias com o solo no depósito de lixo das colônias, pois este poderia estar contaminado com fungos saprofíticos e parasitas (LACERDA et al. 2014). Outros gêneros encontrados podem atuar como competidores do fungo simbionte cultivado por Attini. Algumas espécies do gênero Fusarium têm sido encontradas frequentemente no jardim de fungo de Attini (Rodrigues et al. 2008) e em nosso estudo encontramos Fusarium solani (Martius) Saccardo em operárias.

Outro gênero comumente encontrado no jardim de fungos das cortadeiras é Aspergillus. RodRigues (2009) isolou várias espécies desse gênero em ninhos de Attini e também em rainhas recém-fecundadas de Atta. Apesar de serem consideradas espécies oportunistas, que raramente causam surtos epizoóticos, algumas delas podem se mostrar altamente virulentas em testes de laboratório, como é o caso de Aspergillus ochraceus Wilhelm em operárias de Atta bisphaerica (Forel) (RIBEIRo et al. 2012) ou Aspergillus nomius Kurtzman et al. em operárias de Acromyrmex echinatior (Forel) (Poulsen et al. 2006), por exemplo.

Estudos desenvolvidos por Hughes et al. (2004), mostram que, de 20 ninhos amostrados de quatro espécies de cortadeiras, em 18 desses havia contaminação com o fungo $M$. anisopliae nos próximos $5 \mathrm{~cm}$ de terra ao redor de cada ninho. Porém, poucas operárias se encontravam infectadas. Isso indica que as estratégias de defesa empregadas pelas formigas mostram-se eficientes contra esse fungo. Chama atenção no nosso estudo a taxa de infecção de operárias de $A$. sexdens por $M$. anisopliae. Ele é um fungo entomopatogênico bem estudado e ativo contra muitas espécies de insetos (ZimmermanN 1993). No entanto, surtos epizoóticos devido a esse fungo, em colônias do campo, não têm sido relatados, a despeito de sua proximidade aos ninhos e ocorrência em operárias forrageadoras (Hughes et al. 2004). Por outro lado, em laboratório, demonstrou-se que altas doses desse fungo provocaram a morte das operárias médias de $A$. sexdens, sendo estas as que tentaram efetuar a limpeza do ninho (JACCOUD et al. 1999).

A busca de fungos entomopatogênicos associados às formigascortadeiras, bem como fungos antagonistas do seu simbionte mutualista é importante para fornecer subsídio para um futuro controle microbiano das espécies-praga. Em testes de laboratório, um isolado de $M$. anisopliae se mostrou altamente virulento às operárias de $A$. sexdens, o que encoraja a busca de formas de se viabilizar o uso desses agentes entomopatogênicos no controle de colônias do campo.

\section{AGRADECIMENTOS}

Os autores agradecem à equipe do Dr. André Rodrigues (UNESPRio Claro) pelo auxílio na identificação dos fungos e ao $\mathrm{CNPq}$ pelo suporte financeiro (Processo 403708-2013-3)

\section{REFERÊNCIAS}

Alexopoulos, C.J., C.W. Mims \& M. Blackwell, 1996. Introductory Mycology. Hoboken (NJ), John Wiley \& Sons, 868 p.

Alves, R.T. \& M. Faria, 2010. Pequeno manual sobre fungos entomopatogênicos. Planaltina (DF), Embrapa Cerrados, $5^{0}$ p.

Barnett, H.L. \& B.B. Hunter, 1998. Illustrated genera of imperfect fungi. St. Paul (MN), American Phytopathological Society, $240 \mathrm{p}$.

Breen, J.P., 1994. Acremonium endophyte interactions with enhanced plant resistance to insects. Annual Review of Entomology, 39: 401-423. DOI: http://dx.doi.org/10.1146/annurev.en.39.010194.002153.

Della Lucia, T.M.C., L.C. Gandra \& R.N.C. Guedes, 2014. Managing leaf-cutting ants: peculiarities, trends and challenges. Pest Management Science, 70: 14-23. DOI: http://dx.doi.org/10.1002/ps.3660.
Diehl-Fleig, E., M.E. Silva \& M.R.M. Pacheco, 1988. Testes de patogenicidade dos fungos entomopatogênicos Beauveria bassiana e Metarhizium anisopliae em Atta sexdens piriventris (Santsch., 1919) em diferentes temperaturas. Ciência e Cultura, 40: 1103-1105.

Domsch, K.H., W. Gams \& A. Traute-Heidi, 2007. Compendium of soil fungi, $2^{\text {nd }}$ Ed. Eching (Alemanha), IHW-Verlag \& Verlagsbuchhandlung, $672 \mathrm{p}$.

Ferraz, R.E., P.M. Lima, D.S. Pereira, C.C.O. Freitas \& F.M.C. Feijó, 2008. Microbiota fúngica de Melipona subnitida Ducke (Hymenoptera: Apidae). Neotropical Entomology, 37: 345-346. DOI: http://dx.doi.org/10.1590/S1519566X2008000300017.

Finney, D.J., 1952. Probit analysis, New York, Cambridge University Press, $318 \mathrm{p}$.

Grandeza, L.A.O., J.C. Moraes \& R. Zanetti, 1999. Estimativa do crescimento externo de ninhos de Atta sexdens rubropilosa Forel e Atta laevigata (F. Smith) (Hymenoptera: Formicidae) em áreas de reflorestamento com eucalipto. Anais da Sociedade Entomológica do Brasil, 28: 59-64. DOI: http://dx.doi.org/10.1590/So301-80591999000100006.

Hughes, W.O. \& J.J. Boomsma, 2004. Let your enemy do the work: within-host interactions between two fungal parasites of leaf-cutting ants. Proceedings of Royal Society of London, B, Biological Sciences, 271: S104-S106. DOI: http://dx.doi.org/10.1098\%2Frsbl.2003.0115.

Hughes, W.O.H., L. Thomsen, J. Eilenberg \& J.J. Boomsma, 2004. Diversity of entomopathogenic fungi near leafcutting ant nests in a Neotropical forest, with particular reference to Metarhizium anisopliae var. anisopliae. Journal of Invertebrate Pathology, 85: 46-53. DOI: http://dx.doi.org/10.1016/i.jip.2003.12.005.

Jaccoud, D.B., 2000. Formigas cortadeiras: princípios de manejo integrado de áreas infestadas. Brasília, IBAMA, 60 p.

Jaccoud, D.B., W.O.H. Hugues \& C.W. Jackson, 1999. The epizootiology of a Metarhizium infection in mini-nests of the leaf-cutting ant Atta sexdens rubropilosa. Entomologia Experimentalis et Applicata, 93: 51-61, 1999. DOI: http://dx.doi.org/10.1046/j.1570-7458.1999.00561.x.

Kaplan, E.L. \& P. Meier, 1958. Nonparametric estimation from incomplete observations. Journal of the American Statistical Association, 53: 457-481.

Klingenberg, C. \& C.R.F. Brandão, 2009. Revision of the fungus-growing ant genera Mycetophylax Emery and Paramycetophylax Kusnezov rev. stat., and description of Kalathomyrmex n. gen. (Formicidae: Myrmicinae: Attini). Zootaxa, 2052: 1-31.

Lacerda, F.G., T.M.C. Della Lucia, O. DeSouza, O.L. Pereira, M.C.M. Kasuya, L.M. de Souza, J.C. Couceiro \& D.J. de Souza, 2014. Social interactions between fungus garden and external workers of Atta sexdens (Linnaeus) (Hymenoptera: Formicidae). Italian Journal of Zoology, 81: 298-303. DOI: http://dx.doi.org/10.1080/11250003.2014.911369.

Lopez, E. \& S. Orduz, 2003. Metarhizium anisopliae and Trichoderma viride for control of nests of the fungus-growing ant, Atta cephalotes. Biological Control, 27: 194-200. DOI: http://dx.doi.org/10.1016/S1049-9644(03)00005-7.

Mantel, N., 1966. Evaluation of survival data and two new rank order statistics arising in its consideration. Cancer Chemotherapy Reports, 50: 163-170.

Maurya, P., L. Mohan, P. Sharma \& C.N. Srivastava, 2011. Evaluation of larvicidal potential of certain insect pathogenic fungi extracts against Anopheles stephensi and Culex quinquefasciatus. Entomological Research, 41: 211-215. DOI: http://dx.doi.org/10.1111/j.1748-5967.2011.00347.x.

Peto, R. \& J. Peto, 1972. Asymptotically efficient rank invariant test procedures. Journal of the Royal Statistical Society (Series A), 135: 185-207. DOI: http://dx.doi.org/10.2307/2344317.

Poulsen, M., W.O.H. Hughes \& J.J. Boomsma, 2006. Differential resistance and the importance of antibiotic production in Acromyrmex echinatior leaf-cutting ant castes towards the 
entomopathogenic fungus Aspergillus nomius. Insectes Sociaux, 53: 349-355. DOI: http://dx.doi.org/10.1007/ s00040-006-0880-y.

Ribeiro, M.R., K.D. Amaral, V.E. Seide, B.M.R. Souza, T.M.C. Della Lucia, M.C.M. Kasuya \& D.J. de Souza, 2012. Diversity of fungi associated with Atta bisphaerica (Hymenoptera: Formicidae): The activity of Aspergillus ochraceus and Beauveria bassiana. Psyche, Article ID 389806, 6 p. DOI: http://dx.doi.org/10.1155/2012/389806.

Rodrigues, A., 2009. O papel dos microfungos associados aos jardins das formigas Attini (Hymenoptera: Formicidae).Tese (Doutorado em Ciências Biológicas: Microbiologia Aplicada) - Universidade Estadual Paulista. 148 p.

Rodrigues, A., M. Bacci Jr., U. G. Mueller, A.Ortiz \& F.C. Pagnocca, 2008. Microfungal "weeds" in the leafcutter ant symbiosis. Microbial Ecology, 56: 604-614. DOI: http://dx.doi.org/10.1007/s00248-008-9380-0.

Rodriguez, R.J., J.F. White Jr., A.E. Arnold \& R.S. Redman, 2009. Fungal endophytes: diversity and functional roles. New Phytologist, 182: 314-330. DOI: http://dx.doi.org/10.1111/ j.1469-8137.2009.02773.x.

Samson, R.A., 2000. Introduction to food and airborne fungi. Washington, American Society for Microbiology, 389 p.

Schultz, T.R. \& S.G. Brady, 2008. Major evolutionary transitions in ant agriculture. Proceeding of National Academy of Sciences of USA, 105: 5435-5440. DOI: http://dx.doi.org/10.1073/ pnas.0711024105.

Sosa-Calvo, J., T.R. Schultz, C.R.F. Brandão, C. Klingenberg, R.M.Feitosa, C. Rabeling, M. Bacci Jr., C.T. Lopes \& H.L. Vasconcelos, 2013. Cyatta abscondita: taxonomy, evolution, and natural history of a new fungus-farming ant genus from Brazil. PLoS ONE, 8: e80498. DOI: http://dx.doi.org/10.1371/journal.pone.0080498.

Statsoft, 2004. STATISTICA (data analysis software system), versão 7. Disponível em: <http://www.statsoft.com $>$.

Wilson, E.O., 1971. The insect societies. Cambridge, Harvard University Press, 562 p.

Zimmermann, G., 1993. The entomopathogenic fungus Metarhizium anisopliae and its potential as a biocontrol agent. Pesticide Science, 37: 375-379. DOI: http://dx.doi.org/10.1002/ps.2780370410.

\section{Recebido em: 05.v.2015}

Aceito em: 10.xii.2015

$* * * * * * * * *$

\section{Como citar este artigo:}

Dornelas, A.S.P., R.A. Sarmento, G.R. dos Santos, M.O. Nascimento \& D.J. de Souza, 2016. Fungos Filamentosos Associados às Espécies Atta sexdens (Linnaeus) e Atta laevigata (F. Smith) (Hymenoptera: Formicidae). EntomoBrasilis, 9 (1): 26-30.

Acessível em: doi:10.12741/ebrasilis.vgi1.528
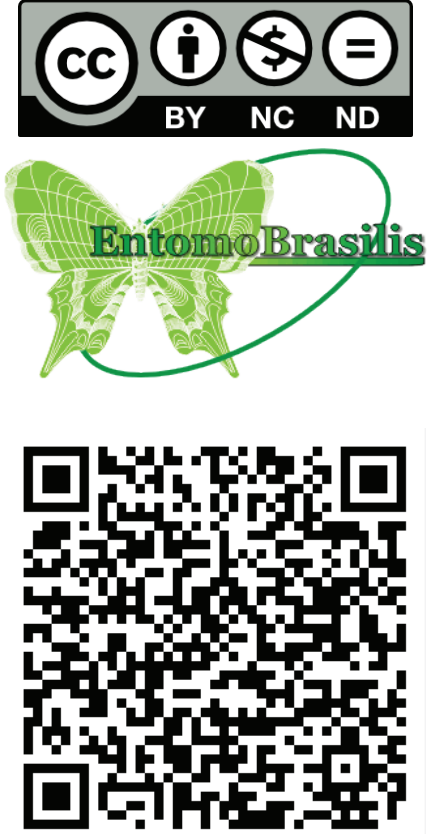